\title{
QUANTIFICATION OF SENSORY INFORMATION IN HUMAN BALANCE CONTROL
}

\author{
Herman van der Kooij*, Bart Koopman*, Ron Jacobs**, Thomas Mergner***, Henk Grootenboer* \\ *Institute of Biomedical Technology (BMTI), University of Twente, Enschede, The Netherlands \\ ** Intelligent Inference Systems Corp., Sunnyvale CA, USA \\ *** Neurologische Klinik, Neurozentrum, Freiburg, Germany \\ *E-mail: h.vanderkooij@wb.utwente.nl
}

\begin{abstract}
A human balance control model is developed, which includes the different sensory systems as well as neural time delays. The model is based on optimal control theory. Platform perturbation experiments were done to quantify the precision of the different sensory systems by matching model predictions with experimental results. The precision of the sensors was quantified by the variances of sensor noise. The noise to signal ratios for the muscle spindles are 3-7\% and for vision $11-14 \%$. For the vestibular organs unambiguous noise to signal ratios could not be found. To find the noise to signal ratios of the vestibular organs the method of identification of sensory information has to be modified.
\end{abstract}

\section{INTRODUCTION}

It is known from human balance experiments that altered or ambiguous sensory input conditions affect balance [1]. Existing models of human balance control [2] ignore the problem of neural time delays and all the different sensory systems humans utilize in maintaining balance. Therefore, these models could not address the question how multi sensory information is integrated.

In this paper, a model is presented, which includes the different sensory systems as well as neural time delays. In the model, all sensory information is integrated to make an optimal estimate of body orientation. The weighting of the different sensory input depends on the precision of the sensors. To determine the precision of each sensory system, experiments were performed in which subjects had to maintain balance while standing on a moving platform. By matching experimental results with model predictions, the precision of the sensors was quantified.

\section{METHODS}

\section{A. Subjects and experimental procedure}

Four male subjects (age $31 \pm 5 \mathrm{yr}$. , body mass $86.5 \pm 9.8 \mathrm{~kg}$., body height $1.83 \pm 0.05 \mathrm{~m}$., leg length $0.92 \pm 0.14 \mathrm{~m}$.) participated in this study. The subjects were instructed to stand as still as possible on a $6 \mathrm{DOF}$ movable platform (a hexapod) for $200 \mathrm{~s}$. each trial. The study consisted of eight trials with four different random platform movements in the sagittal plane (table 1) with eyes open (EO) and blindfolded
(EC). Platform rotations and translations were generated by low-pass filtering a random signal with a second order Butterworh filter and limited to \pm 3.5 degrees and $\pm 6 \mathrm{~cm}$ respectively. With eyes open the subjects had to look at a structured visual scene, generated by a $3 D$ virtual environment program. Headphones were worn to exclude auditory clues from the platform. During the execution of the task, the movement patterns and the ground-reaction forces were recorded with a sample frequency of $100 \mathrm{~Hz}$.

\begin{tabular}{|l||lr|ll|}
\hline Platform movement & \multicolumn{2}{|l|}{ Slow(S) } & \multicolumn{2}{l|}{ fast(F) } \\
\hline Rotation only (R) & 1) & $0-0.03 \mathrm{~Hz}$ & 2) & $0-0.10 \mathrm{~Hz}$ \\
\hline Rotation and & $3)$ & $0-0.03 \mathrm{~Hz}$ & \multirow{2}{*}{ 4) } & $0-0.10 \mathrm{~Hz}$ \\
translation (R+T) & 3) & $0-0.05 \mathrm{~Hz}$ & & $0-0.20 \mathrm{~Hz}$ \\
\hline
\end{tabular}

Table 1: Overview of platform movements and their cut-off frequencies. $R$ is platform rotation, $R+T$ is simultaneously rotation and translation. The platform speed was either fast (F) or slow (S.)

\section{B. Kinematics and treatment of the data}

Landmark coordinates (Figure 1A) were recorded with the aid of a motion analyzer (Optotrack) and band-pass filtered with a second order Butterworth filter $(.05-3 \mathrm{~Hz})$. These coordinates were used to calculate the angles of three body segments in the sagittal plane (Figure 1B). For each time series of segment angles, the variance was calculated. Second and third derivatives of horizontal platform translations and second derivatives of platform rotations were computed numerically. The variance of the time series of these derivatives was also calculated.
A)

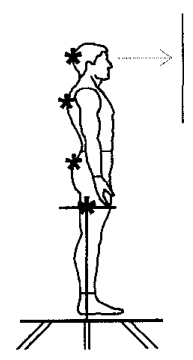

B)

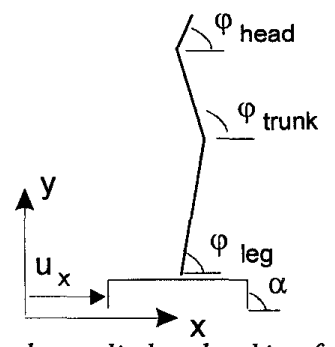

Fig 1: A) positions of landmarks applied to the skin of the subject and to the movable platform. B) Segment model, consisting of a leg, trunk and head 


\section{Human Stance Control Model}

It is assumed that humans maintain balance in an optimal way. A stance control model is developed based on this principle (Figure 2). A standing person on a movable platform is modeled by a three-link segment model in the sagittal plane (Figure 1B). The three segments represent the legs, trunk and head. The segments are connected by friction-free hinge joints. The muscles are modeled as torque actuators at the joint. The sensor dynamics of muscle spindles, skin afferents in the sole, vision, semi-circular canals and otoliths are approximated by transfer functions obtained from literature [3]. It is assumed that a best possible estimate of human body orientation is made in the sensory integration center. The sensory integration center is a combination of an extended Kalman filter in combination with a predictive element, which compensates for neural time delays, which are in the order of $100 \mathrm{~ms}$. The action control center selects muscle actions to minimize the difference between the estimated and desired body orientation in an optimal way. This is realized by a Linear Quadratic Regulator (LQR), which minimizes the cost function

$$
J=\int_{0}^{\infty}\left(\left(\underline{\hat{x}}_{s t}-\underline{x}_{r e f}\right)^{T} Q\left(\underline{\hat{x}}_{s t}-\underline{x}_{r e f}\right)+\underline{u}_{s t}^{T} R \underline{u}_{s t}\right) d t
$$

where $R$ and $Q$ are weighting matrices, penalizing muscle actions and deviations from the desired body orientation respectively. The model is described in more detail elsewhere [4]

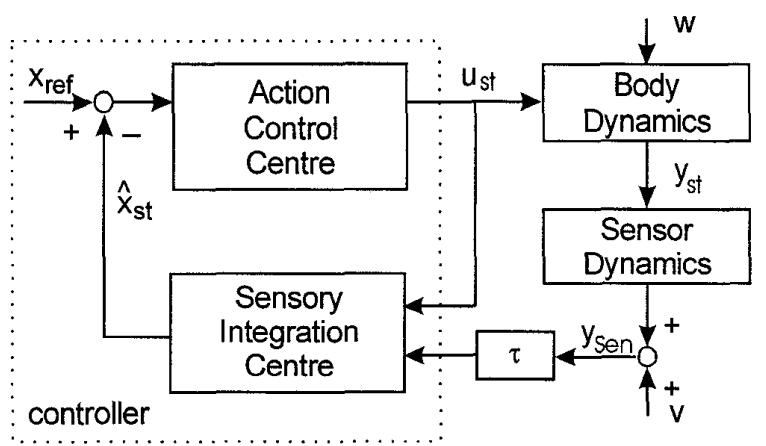

Figure 2.: Flow diagram of the human stance control model. Body dynamics describes a standing person controlled by muscle actions $\left(u_{s \downarrow}\right)$ and exposed to unknown perturbations $\left(w_{s}\right)$. Sensor dynamics relates the sensory input $\left(y_{s}\right)$ to the sensory output $\left(y_{\text {ser }}\right)$. The delayed sensory output afflicted with noise (v) and the muscle actions are input to the sensory integration Centre which makes a best estimate of body orientation $\left(x_{s}\right)$. Based on this estimate the action control centre selects muscle actions in order to maintain standing and to achieve a desired orientation of the body $\left(x_{\text {ref }}\right)$.

By linearizing the human stance control model around the desired orientation, a closed form expression for the variance of the segment angles can be obtained [5]. This expression is a function of:

1. Modeled body and sensor dynamics

2. Neural time delay of $100 \mathrm{~ms}$.

3. Computed variances of second and third derivatives of horizontal platform translations and of the second derivative of platform rotations.

4. Unknown weights in the objective function of the LQR

5. Unknown variance's of the noise afflicting the sensory output of the modeled sensory systems $\left(V=E\left\{v^{T} v\right\}\right)$

\section{Identification of the unknown model input parameters}

The unknown model parameters were found by means of the Goal Attainment Method of Gembicki [6]. This optimization method tries to makes the objective function

$$
F(x)=\left\{F_{i}(x), F_{2}(x), \ldots, F_{m}(x)\right\}
$$

attain the goal,

$$
F^{*}=\left\{F_{1}^{*}, F_{2}^{*}, \ldots, F_{m}^{*}\right\}
$$

by varying $\mathrm{x}$. Define $F^{*}$ as a vector with the $m$ experimental found variances of the segment angles for the different experimental conditions, $F(x)$ as a vector with $m$ closed form expressions for the variance of the segment angles for different conditions and $\mathrm{x}$ is a vector with the unknown weights in the objective function of the $L Q R$ and the unknown variance of the sensor noise $(V)$. These unknown model parameters were found by solving the above optimization problem. To reduce the complexity of the optimization problem the following assumptions were made:

1. The variance's of the sensor noise of the muscle spindles acting around the ankle, hip and neck are identical.

2. The weighting matrix $(Q)$, penalizing deviations from the desired body orientation, equals the identity matrix

3. The weighting matrix $(R)$, penalizing muscle actions, is a diagonal matrix which entries are unknown

As a consequence of above assumptions, $x$ is a vector of length 8 , containing the sensor noise variance's of the muscle spindles, the skin afferents in the sole, the semicircular canals, the otoliths and the visual system and the weights penalizing muscle actions around the ankle, hip and neck. The variance of the sensor noise can be expressed as [7]:

$$
V=\frac{P_{0} E\left\{y^{T} y\right\}}{K^{2}\left(a, \sigma_{y}\right)}
$$

Where $P_{0}$ is the noise to signal ratio and $\mathrm{K}$ equals one when the sensory output is always above its threshold $a$ and varies between zero and one when the sensory output is always or partially below its threshold. The found variances of the sensor noise are expressed in their noise to signal ratio $P_{0}$. To express how well the model predictions resemble the measured segment angles after optimization a score is calculated, defined by: 


$$
\text { score }=\sum_{i=1, \ldots m}\left(\frac{F_{i}(x)-F_{i}^{*}}{F_{i}^{*}}\right)^{2}
$$

\section{RESULTS}

\section{A. Kinematics}

Table 2 shows the calculated variances of the leg, trunk and head angle of the different subjects for the eight experimental conditions. For the same platform movements, the variances of the segment angles are larger for $\mathrm{EC}$ than for $\mathrm{EO}$. Comparison of the results of different types of platform movements ( $R$ and $R+T$ ), with the same other experimental conditions, shows that the variances of the segment angles are larger for $\mathrm{R}+\mathrm{T}$ than for $\mathrm{R}$. Above-mentioned goes for all subjects except for subject 3 and subject 4 with eyes closed (EC). Comparison of the results of slow and fast platform movements, with the same other experimental conditions, does not show a clear tendency. Differences between subjects depend on experimental conditions.

\begin{tabular}{|c|c|c|c|c|c|c|c|c|}
\hline \multicolumn{9}{|c|}{ Subject $1(\mathrm{~S} 1)$} \\
\hline & \multicolumn{4}{|c|}{ Slow } & \multicolumn{4}{|c|}{ Fast } \\
\hline & \multicolumn{2}{|c|}{$\mathrm{R}$} & \multicolumn{2}{|c|}{$\mathrm{R}+\mathrm{T}$} & \multicolumn{2}{|c|}{$\mathrm{R}$} & \multicolumn{2}{|c|}{$\mathrm{R}+\mathrm{T}$} \\
\hline & EO & $\mathrm{EC}$ & EO & $\mathrm{EC}$ & $\mathrm{EO}$ & $\overline{\mathrm{EC}}$ & EO & $\overline{\mathrm{EC}}$ \\
\hline legs & 0.40 & 1.22 & 0.92 & 1.25 & 0.57 & 1.42 & 1.12 & 2.01 \\
\hline trunk & 0.17 & 0.70 & 0.43 & 0.91 & 0.19 & 0.98 & 0.46 & 1.53 \\
\hline head & 0.47 & 1.54 & 0.86 & 2.06 & 0.71 & 1.86 & 0.72 & 2.34 \\
\hline \multicolumn{9}{|c|}{ Subject 2 (S2) } \\
\hline & \multicolumn{4}{|c|}{ Slow } & \multicolumn{4}{|c|}{ Fast } \\
\hline & \multicolumn{2}{|c|}{$\mathrm{R}$} & \multicolumn{2}{|c|}{$\mathrm{R}+\mathrm{T}$} & \multicolumn{2}{|c|}{$\mathrm{R}$} & \multicolumn{2}{|c|}{$\mathrm{R}+\mathrm{T}$} \\
\hline & $\mathrm{EO}$ & EC & EO & $\mathrm{EC}$ & EO & $\mathrm{EC}$ & EO & $\mathrm{EC}$ \\
\hline legs & 0.36 & 0.89 & 0.39 & 0.54 & 0.53 & 0.89 & 0.79 & 0.80 \\
\hline trunk & 0.16 & 0.41 & 0.16 & 0.49 & 0.17 & 0.38 & 0.29 & 0.78 \\
\hline head & 0.36 & 0.46 & 0.48 & 0.77 & 0.28 & 0.39 & 0.37 & 0.82 \\
\hline \multicolumn{9}{|c|}{ Subject 3 (S3) } \\
\hline & \multicolumn{4}{|c|}{ Slow } & \multicolumn{4}{|c|}{ Fast } \\
\hline & \multicolumn{2}{|c|}{$\mathrm{R}$} & \multicolumn{2}{|c|}{$\mathrm{R}+\mathrm{T}$} & \multicolumn{2}{|c|}{$\mathrm{R}$} & \multicolumn{2}{|c|}{$\mathrm{R}+\mathrm{T}$} \\
\hline & EO & $\mathrm{EC}$ & EO & $\mathrm{EC}$ & EO & $\mathrm{EC}$ & EO & $\mathrm{EC}$ \\
\hline legs & 0.45 & 0.96 & 0.50 & 0.56 & 0.48 & 1.63 & 0.83 & 1.15 \\
\hline trunk & 0.20 & 0.96 & 0.32 & 0.61 & 0.16 & 0.92 & 0.27 & 0.69 \\
\hline head & 0.28 & 1.06 & 0.36 & 0.77 & 0.18 & 1.00 & 0.29 & 0.86 \\
\hline \multicolumn{9}{|c|}{ subject $4(\mathrm{~S} 4)$} \\
\hline & \multicolumn{4}{|c|}{ Slow } & \multicolumn{4}{|c|}{ Fast } \\
\hline & \multicolumn{2}{|c|}{$\mathrm{R}$} & \multicolumn{2}{|c|}{$\mathrm{R}+\mathrm{T}$} & \multicolumn{2}{|c|}{$\mathrm{R}$} & \multicolumn{2}{|c|}{$\mathrm{R}+\mathrm{T}$} \\
\hline & $\mathrm{EO}$ & $\mathrm{EC}$ & EO & $\mathrm{EC}$ & EO & $\overline{\mathrm{EC}}$ & EO & $\overline{\mathrm{EC}}$ \\
\hline legs & 0.41 & 1.06 & 0.69 & 1.22 & 0.51 & 1.96 & 0.88 & 1.95 \\
\hline trunk & 0.17 & 0.59 & 0.29 & 0.57 & 0.28 & 0.77 & 0.43 & 0.98 \\
\hline head & 0.33 & 0.76 & 0.32 & 0.87 & 0.32 & 1.01 & 0.64 & 1.20 \\
\hline
\end{tabular}

Table 2.: measured variances of the segment angles in $\mathrm{deg}^{2}$ of the different subjects, while standing on a moving platform under different conditions. $R$ is platform rotation, $R+T$ is simultaneously rotation and translation. The platform speed was either fast or slow. The subjects had their eyes open (EO) or were blindfolded (EC).

\section{B. Identification of the unknown model input parameters}

It was not possible to find one unique vector $x$, which could predict all experimental, found variances of the segment angles. Therefore, the vector $\mathrm{F}^{*}$ of length 24 was converted to a set of vectors of length $6, \mathrm{~F}^{* *}$. Different forms of $\mathrm{F}^{* *}$ were examined, by grouping the experimental found variances of the segment angles, for the same:

A. Type of platform movement and platform speed

B. Visual condition and platform speed.

C. Type of platform movement and visual condition

Each type of grouping results in a subset of four vectors $\mathrm{F}^{* *}$ (table 3 ). The unknown parameters, lumped in the vector $\mathrm{x}$, which gave the best match between $F(x)$ and $F^{* *}$ were found by optimization. For different starting values of $x$, a unique solution of $\mathrm{x}$ was found.

\begin{tabular}{|l|c|c|c|c|c|c|c|c|}
\hline \multirow{2}{*}{ grouping } & \multicolumn{4}{|c|}{ Slow } & \multicolumn{4}{c|}{ Fast } \\
\cline { 2 - 9 } & \multicolumn{3}{|c|}{ R } & \multicolumn{2}{|c|}{ R+T } & \multicolumn{3}{|c|}{ R+T } \\
\cline { 2 - 9 }$y$ & EO & EC & EO & EC & EO & EC & EO & EC \\
\hline A & A1 & A1 & A2 & A2 & A3 & A3 & A4 & A4 \\
\hline B & B1 & B2 & B1 & B2 & B3 & B4 & B3 & B4 \\
\hline C & C1 & C2 & C3 & C4 & C1 & C2 & C3 & C4 \\
\hline
\end{tabular}

Table 3.: Overview of grouping the experimentally found variances of the segment angles. Each type of grouping (A$C)$ yields four subsets with variances of the segment angles.

Grouping the experimental results for the same visual condition and platform speed (B) yielded the best match between model predictions and experimental results, expressed in the calculated score (tabel 4). The found variances of the noise are summarized in table 4 . For the found variances of signal noise, the noise to signal ratios of the muscle spindles and the visual system were calculated (table 4). The calculated noise to signal ratios of the vestibular system (not shown) differed very much for the different experimental conditions. The noise to signal ratio of the sole afferents was very high, which indicates that in the model the sole afferents do not contribute to balance. Removal of the sole afferents in the model did not effect the model predictions. The noise to signal ratios of the muscle spindles are all in the range of 3-7\%. For vision the noise to signal ratios varies from $11-14 \%$, except for subject 5 in case of $B 1$. The variance of the otolith signal noise varies between 0.22 and 4.78. The variance of the semi-circular signal noise varies between 0.03 and 1.56. A sensitivity analysis showed that model predictions are quite insensitive for changes in the variance of the semi-circular signal noise. Model predictions are very sensitive for changes in the variance of the otolith signal noise. 


\begin{tabular}{|c|c|c|c|c|c|c|c|}
\hline \multirow{2}{*}{ S 1} & \multirow{2}{*}{ score } & \multicolumn{2}{|c|}{ spindles } & canals & otoliths & \multicolumn{2}{|c|}{ vision } \\
\hline & & $V \square]$ & $P_{0}$ & $V[]$ & $V[]$ & $V[]$ & $P_{0}$ \\
\hline B1 & 0.17 & $2.5 \mathrm{e}-5$ & $7.0 \%$ & 1.56 & 0.22 & $1.2 \mathrm{e}-5$ & $13 \%$ \\
\hline B2 & 0.07 & $6.2 e-5$ & $4.3 \%$ & 0.45 & 2.88 & & \\
\hline B3 & 0.31 & $1.9 e-5$ & $4.4 \%$ & 1.56 & 0.33 & $1.4 \mathrm{e}-5$ & $14 \%$ \\
\hline$\overline{\mathrm{B} 4}$ & 0.02 & $5.0 \mathrm{e}-5$ & $5.4 \%$ & 1.56 & 0.76 & & \\
\hline $\mathbf{S 2}$ & & & & & & & \\
\hline$\overline{\mathrm{B} 1}$ & 0.02 & $4.1 \mathrm{e}-6$ & $4.5 \%$ & 1.56 & 0.44 & $3.6 \mathrm{e}-5$ & $11 \%$ \\
\hline$\overline{\mathrm{B} 2}$ & 0.25 & $2.6 \mathrm{e}-5$ & $5.7 \%$ & 1.24 & 0.95 & & \\
\hline B3 & 0.05 & $1.3 \mathrm{e}-5$ & $4.4 \%$ & 0.03 & 0.29 & $1.4 \mathrm{e}-5$ & $14 \%$ \\
\hline$\overline{\mathrm{B} 4}$ & 0.02 & $6.1 e-6$ & $3.3 \%$ & 0.03 & 1.38 & & \\
\hline S 3 & & & & & & & \\
\hline B1 & 0.02 & $4.0 \mathrm{e}-6$ & $4.4 \%$ & 0.07 & 0.60 & $1.0 \mathrm{e}-5$ & $15 \%$ \\
\hline B2 & 0.29 & $1.1 \mathrm{e}-5$ & $4.7 \%$ & 1.56 & 2.88 & & \\
\hline$\overline{B 3}$ & 0.06 & $1.9 \mathrm{e}-5$ & $4.9 \%$ & 1.56 & 0.23 & $1.1 \mathrm{e}-5$ & $13 \%$ \\
\hline $\mathrm{B} 4$ & 0.10 & $3.9 \mathrm{e}-5$ & $5.4 \%$ & 0.03 & 0.72 & & \\
\hline$S 4$ & & & & & & & \\
\hline $\mathrm{B} 1$ & 0.23 & $5.0 e-6$ & $4.9 \%$ & 0.10 & 0.76 & $1,0 \mathrm{e}-6$ & $7.3 \%$ \\
\hline $\mathrm{B} 2$ & 0.17 & $4.8 \mathrm{e}-6$ & $4.4 \%$ & 0.04 & 2.19 & & \\
\hline B3 & 0.01 & $3.2 e-5$ & $4.9 \%$ & 0.00 & 0.26 & $1.1 \mathrm{e}-5$ & $14 \%$ \\
\hline$\overline{\mathrm{B} 4}$ & 0.00 & $2.8 \mathrm{e}-5$ & $4.6 \%$ & 0.16 & 4.78 & & \\
\hline
\end{tabular}

Table 4: The found noise variance's (V) for the modeled muscle spindles, the semi-circular canals, the otoliths and the visual system. These model parameters were found by optimization, minimizing the difference between model predicted and experimental found variance of the segment angles, grouped for the same visual condition and platform speed (type B). For the muscle spindles and vision the noise to signal ratios $\left(P_{o}\right)$ are given. The score is a measure for how well model predictions resemble the measured segment angles. If the score is zero, model predictions and experimental results coincide.

\section{DISCUSSION}

Platform perturbation experiments were done to quantify the precision of the different sensory systems by matching model predictions with experimental results. The precision of the sensors was quantified by the variances of sensor noise. One set of variances of the sensor noise, for which model predictions matched all experimental results, was not found. Different sets of variances of sensor noise were found for which the model predictions matched the results of the experiments with the same visual conditions and platform speed. For the muscle spindles and the visual system, the noise to signal ratios are in reasonable small ranges. The vestibular noise to signal ratios differed very much for the various experimental conditions.

At this moment, quantification of the precision of the vestibular signals by their noise to signal ratio is not possible. An explanation might be that in the optimization process the variances of the vestibular sensor noise are kept constant for different experimental conditions. This could be unrealistic since in the model the variances of the vestibular sensor noise strongly vary due to the thresholds of the vestibular organs.
A different explanation might be the assumption that the control strategy does not change under different perturbation conditions and different visual conditions. When matching model predictions and experimental results by optimization, the weights penalizing muscle actions around the knee, hip and ankle are kept constant

To overcome these problems, the method of identifying unknown model parameters has to be modified. The variances of the vestibular signal noise and the control strategies should not be kept constant for the different experimental conditions. This modification could however lead to computational problems and could complicate the finding of unique solutions, since the set of model parameters becomes too large. It might be necessary to reduce the set of model parameters. This could be realized by experiments where subjects are acting like an inverted pendulum. This could be achieved by choosing the perturbations properly or by mechanically locking for example the knee and hip joint. These subjects need further investigation.

\section{CONCLUSION}

Platform perturbation experiments were done to quantify the precision of the different sensory systems by matching model predictions with experimental results. The precision of the sensors was quantified by the variances of the sensor noise. The noise to signal ratios for the muscle spindles are $3-7 \%$ and for vision $11-14 \%$. For the vestibular organs unambiguous noise to signal ratios could not be found. To find the noise to signal ratios of the vestibular organs the method of identifying sensory information has to be modified

\section{REFERENCES}

[1] F. B. Horak and J. M. Macpherson, "Postural orientation and equilibrium," in Exercise: regulation and integration of multiple systems., L. Rowell and J. Saheppard, Eds. New York:Oxford University Press, 1996

[2] A. D. Kuo, "An optimal control model for analyzing human postural balance," IEEE Trans. Biom. Eng., vol. 42, pp. 87-101, 1995.

[3] J. L. Borha, R.Young and R. F. Curry, "Optimal estimator for human spatial orientation," Ann. NY. Acad. Sci., vol. 545, pp. 51-73, 1988.

[4] H. van der Kooij, R. Jacobs, H. F. J. M. Koopman and H. J. Grootenboer, "Model to quantify mulit-sensory information in postural control," Bio. Cyber. (submitted), 1997.

[5] D. L. Kleinman, "Optimal control of linear systems with time-delay and observation noise," IEEE Trans Automat. Control, vol. 15, pp. 524 527,1969 .

[6] F. W. Gembicki, "Vector optimization for control with performance and Parameters sensitive indices," Ph.D. dissertation., , Case Western Reserve Univ :Cleveland, Ohio, 1974

[7] P. H. Weverink, "Models of the human observer and controller of a dynamic system,", Ph.D. dissertation, University of Twente; Enschede, The Netherlands, 1989 